J. Clin. Chem. Clin. Biochem.

Vol. 16, 1978, pp. 145-149

\title{
Gaschromatographische Bestimmung von Di- und Trisacchariden ${ }^{1}$ )
}

\author{
Von H. Schwind, F. Scharbert, R. Schmidt und R. Kattermann \\ Klinisch-Chemisches Institut (Dir.: Prof. Dr. R. Kattermann) Klinikum Mannheim der Universität Heidelberg
}

(Eingegangen am 18. Mai 1977)

Zusammenfassung: Es werden drei neue Verfahren zur gaschromatographischen Trennung von Di- bzw. Trisacchariden angegeben.

Methode I: Methoximbildung mittels Methoxylaminhydrochlorid in Pyridin und anschließende Veresterung mit Acetanhydrid. Trennung auf einer OV 225 -Säule bei $260^{\circ} \mathrm{C}$.

Methode II: Reduktion der Disaccharide zu den Alditolen mit Natriumborhydrid in wäßriger Lösung und anschlieBende Acetylierung mit Acetanhydrid in Pyridin. Bei diesem Verfahren entsteht für jeden Zucker nur ein einzelner Peak. Trennung auf einer OV 225-Säule bei $260^{\circ} \mathrm{C}$.

Methode III: Überführung der Zucker in die Methoxime mittels Methoxylaminhydrochlorid in Pyridin und anschlieBende Trifluoracetylierung mit N-Methyl-bis-(trifluoracetamid). Die Derivate der Trisaccharide sind noch ausreichend flüchtig. Trennung auf einer OV 101 -Säule bei $130^{\circ} \mathrm{C}$ für die Disaccharide und $160^{\circ} \mathrm{C}$ für die Trisaccharide.

Die Retentionszeiten biologisch vorkommender Di- und Trisaccharide wie Maltose, Maltotriose, Lactose und Saccharose werden mitgeteilt. Am Beispiel der Lactose, Maltose und Saccharose werden die mit Methode I erzielten Zuverlässigkeitskriterien beschrieben.

\section{Gas chromatographic determination of di- and trisaccharides}

Summary: Three new methods are reported for the gas chromatographic analysis of di- and trisaccharides.

Method I: Sugars are converted into their methoximes by reaction with methoxylamine hydrochloride in pyridine, followed by esterification with acetic anhydride. The separation is performed on OV 225 at $260^{\circ} \mathrm{C}$.

Method II: Disaccharides are reduced to the alditols with sodium borohydride in aqueous solution, followed by acetylation with acetic anhydride in pyridine. In this method, only one peak is observed for each sugar. The derivatives are separated on OV 225 at $260^{\circ} \mathrm{C}$.

Method III: Sugars are converted into their methoximes by reaction with methoxylamine hydrochloride in pyridine, followed by trifluoroacetylation with $\mathrm{N}$-methyl-bis (trifluoroacetamide). The trisaccharide derivatives still show sufficient volatility. The separation is performed on OV 101 at $130^{\circ} \mathrm{C}$ for disaccharides, and at $160^{\circ} \mathrm{C}$ for trisaccharides.

The retention times for biologically occurring di- and trisaccharides such as maltose, maltotriose, lactose, and sucrose are reported. The reliability criteria for method I are reported for the analysis of lactose, maltose and sucrose.

\section{Einführung}

Die qualitative Differenzierung und quantitative Bestimmung von Mono=, Di- und Oligosacchariden in biologischen Flüssigkeiten oder Geweben gewinnt in der biochemischen Forschung ein zunehmendes Interesse.

1) Über einen Teil der hier vorgelegten Ergebnisse wurde bereits auf der 10. Tagung der Deutschen Diabetes-Gesellschaft in Braunlage (1976) und auf der Jahrestagung der Deutschen und Niederländischen Gesellschaften für Klinische Chemie in Aachen (1977) vorgetragen.
Aber auch in der klinischen Diagnostik sind verschiedene Einsatzbereiche einer differenzierten Kohlenhydratanalytik zu nennen:

\section{Durchführung von oralen Glucosetoleranztesten}

Für die Durchführung des oralen Glucosebelastungstests wurde 1972 von der Deutschen Diabetes-Gesellschaft die Gabe von $100 \mathrm{~g}$ Glucose als Standarddosis empfohlen. Wegen der besseren Verträglichkeit und der physiologischen Applikationsform ist nach den Untersuchungen

J. Clin. Chem. Clin. Biochem. / Vol. 16, 1978 / No. 2 
von Mehnert et al. (1) die Verwendung standardisierter Oligosaccharidgemische anstelle der reinen Glucoselösung von Vorteil. Die Interpretation solcher Tests muß eine evtl. gestörte Resorption und den Ubertritt von Dioder Oligosacchariden in das Blut bzw. den Urin berücksichtigen. Stoffwechseluntersuchungen im Rahmen dieser Thematik erfordern ein Verfahren, das die Trennung und direkte quantitative Bestimmung von Disacchariden in den verschiedenen Körperflüssigkeiten erlaubt.

\section{Resorptions- oder Perfusionsstudien am Dünndarm}

Erkrankungen des Dünndarms wie tropische Sprue, Cöliakie, intestinale Sklerodermie und ein erworbener oder angeborener Mangel des Dünndarmepithels an $\beta$-Galaktosidase (EC 3.2.1.23), $\alpha$-Glucosidase (EC 3.2.1.20) oder $\beta$-Fructofuranosidase (EC 3.2.1.26) sind durch eine gestörte Resorption der Disaccharide gekennzeichnet. Dies kann daran erkannt werden, daß im Dünndarmperfusat die Konzentration eines Disaccharids langsamer als beim Gesunden abfällt oder Disaccharide durch passive Diffusion durch das Dünndarmepithel ungespalten in das Blut gelangen.

\section{Differenzierung unklarer Melliturien}

Besonders in der Pädiatrie ist immer dann eine differenzierte Kohlenhydratanalytik erforderlich, wenn eine Melliturie nicht durch Glucose, sondern durch ein anderes, unbekanntes Mono- oder Disaccharid verursacht ist.

Als indirektes Verfahren kommt die enzymatische Bestimmung von Di- und Trisacchariden - meist als Differenzmessung - nach hydrolytischer Spaltung zu den Monosacchariden in Frage. Direkte Verfahren sind die Papier-, Dünnschicht-, Gas-, Hochdruckflüssigund Ionenaustauschchromatographie, von denen die beiden ersten jedoch nur als semiquantitativ zu betrachten sind. Im folgenden soll die gaschromatographische Bestimmung von Di- und Trisacchariden beschrieben und über unsere Erfahrungen mit dieser Methode berichtet werden.

\section{Material und Methoden}

Um für die Gaschromatographie geeignete Derivate zu erhalten, wurden neuere Derivatisierungsverfahren für Monosaccharide auf verschiedene $\mathrm{Di}$ - bzw. Trisaccharide übertragen. Den als brauchbar befundenen drei Methoden ist gemeinsam, daß die' Carbonylfunktion der Zucker durch Methoximbildung oder Reduktion aufgehoben und die alkoholischen Hydroxylgruppen verestert werden. Dies hat zur Folge, daß in der Regel für jeden Zucker nur ein Peak, in Ausnahmefällen maximal zwei Peaks, erhalten werden. Im Gegensatz dazu bekommt man bei der erstmals von Sweeley et al. (2) angegebenen Trimethylsilylierung der Di- bzw. Trisaccharide in der Regel unübersichtliche Chromatogramme durch Bildung von Anomeren. Außerdem ist die Hydrolyseempfindlichkeit der Derivatisierungsansätze von Nachteil.

\section{Methode I}

Verfahren mit Methoximacetaten als Derivate für Disaccharide Die von Murphy \& Pennock (3) für Monosaccharide angegebene Methode, bei der die Carbonylgruppe mittels Methoxylaminhydrochlorid in Pyridin in das Methoxim überführt wird und im gleichen Ansatz anschließend die Hydroxylgruppen mit Acetanhydrid vollständig verestert werden, läßt sich auf Disaccharide übertragen. Um sicher zu sein, daß die Derivatisierung definiert und quantitativ verläuft, wurden im Halbmikromaßstab die entsprechenden Derivate für die biologisch wichtigsten Disaccharide Maltose, Lactose und Saccharose präparativ dargestellt, kristallisiert und durch Schmelzpunkt, Elementaranalyse und Massenspektrometrie identifiziert, wobei die von uns angenommene Struktur der drei Derivate bestätigt wurde. Sie zeichnen sich durch Hydrolyseunempfindlichkeit und hohe Thermostabilität aus.

\section{Arbeitsvorschrift}

\section{Derivatisierung}

Von den nach der Methode von Somogyi (4) enteiweißten Zuckerlösungen werden bei Konzentrationen unter $2,92 \mathrm{mmol} / \mathrm{l}$ $1 \mathrm{ml}$, bei Konzentrationen über $2,92 \mathrm{mmol} / 1100 \mu \mathrm{l}$ in ein Spitzkölbchen überführt und nach Zufügen von $100 \mu$ l einer Gentiobioselösung der Konzzentration 2,92 mmol/l als innerèr Standard am Rotationsverdampfer zur Trockne eingedampft (evtl. EthanolBenzol-Gemisch (10 ml $+40 \mathrm{ml}$ ) als Wasserschlepper). Der Rückstand wird mit $500 \mu$ l Methoxylaminhydrochlorid (Serva, Heidelberg) in Pyridin ( $10 \mathrm{~g} / \mathrm{l}$ Pyridin) versetzt und $1 \mathrm{~h}$ im Wasserbad bei $70^{\circ} \mathrm{C}$ temperiert. Danach werden $250 \mu$ l Acetanhydrid zugefügt und wiẹder $1 \mathrm{~h}$ bei $70^{\circ} \mathrm{C}$ erwärmt. Die Lösungsmittel werden am Rotationsverdampfer abgezogen und der trockene Rückstand in $500 \mu \mathrm{l}$ Wasser gelöst. Nach Zufügen von $500 \mu \mathrm{l}$ Chloroform und kräftigem Schütteln wird die Chloroform: phase in ein Injektionsgläschen für die Gaschromatographie (Macherey \& Nagel), das mittels Deckel mit Gummi-Teflonmembran versiegelt werden kann, überführt und im Stickstoffstrom bei $70^{\circ} \mathrm{C}$ eingedampft. Der Rückstand wird in $20 \mu \mathrm{l}$ Choroform gelöst, das Gläschen versiegelt und ein Aliquot zur Injektion in den Gaschromatographen verwandt. Die Probe wird nach Perforation der Membran durch die Mikroliterspritze entnommen. Die Derivate bleiben auch bei wochenlangem Stehen bei Raumtemperatur unverändert.

\section{Gaschromatographie}

Gerät:

Säule:

PERKIN-ELMER F 11 (FID),

Trennflüssigkeit: OV 225 (Applied Science Lab. bzw. SERVA, Heidelberg)

3\% auf Gaschrom Q 100-120 mesh (SERVA, Heidelberg),

Trägergas $\mathrm{N}_{2}: \quad 20 \mathrm{ml} / \mathrm{min}, \mathrm{H}_{2}: 40 \mathrm{ml} / \mathrm{min}$, Luft: $400 \mathrm{ml} / \mathrm{min}$,

Säulentemperatur: $260^{\circ} \mathrm{C}$,

Temperatur des

Injektionsblocks: $290^{\circ} \mathrm{C}$,

Temperatur des

FID:

Injektion: $\quad 1 \mu \mathrm{l}$,

Papiervorschub: $\quad 10 \mathrm{~cm} / \mathrm{h}$.

\section{Methode II}

Verfahren mit Alditolacetaten als Derivate für Disaccharide Die Peracetylierung der zuvor mittels Natriumborhydrid in wäßriger Lösung zu den Alditolen reduzierten Monosaccharide stellt ein gängiges gaschromatographisches Verfahren dar, zumal es auch auf Aminozucker angewandt werden kann $(5,6)$. Nachdem sich die peracetylierten Methoxime der Disaccharide bei der zu ihrer gaschromatographischen Trennung erforderlichen hohen Temperatur als ștabil erwiesen hatten, mußte dies auch von den Alditolacetaten der Disaccharide erwartet werden. 


\section{A rbeitsvorschrift}

\section{Derivatisierung}

Von den nach Somogyi (4) enteiweißten Zuckerlösungen wird bei Konzentrationen unter $2,92 \mathrm{mmol} / 11 \mathrm{ml}$, bei Konzentrationen über $2,92 \mathrm{mmol} / 1100 \mu \mathrm{l}$ in ein Spitzkölbchen gegeben und nach Zufügen von $100 \mu 1$ Trehaloselösung der Konzentration $2,92 \mathrm{mmol} / \mathrm{l}$ als innerer Standard mit $300 \mu l$ einer wäßrigen $1 \mathrm{~mol} / 1$ Natriumborhydridlösung versetzt und umgeschüttelt. Der Ansatz bleibt über Nacht stehen. Durch vorsichtige Zugabe von $100 \mu l$ Eisessig wird überschüssiges Natriumborhydrid zerstört. Nach Überführen in ein Injektionsgläschen für die Gaschromatographie wird die Lösung im Stickstoffstrom bei $70^{\circ} \mathrm{C}$ eingedampft (evtl. Ethanol-Benzol-Gemisch $(10 \mathrm{ml}+40 \mathrm{ml})$ als Wasserschlcpper) und der Rückstand dreimal mit je $1 \mathrm{ml}$ eines Methanol-Eiscssig-Gemisches $(200 \mathrm{ml}+1 \mathrm{ml})$ zur Verflüchtigung von Natriumborat als Borsäure trimethylester im Stickstoffstrom abgedampft. Der trockene Rückstand wird mit $500 \mu \mathrm{l}$ Pyridin und $250 \mu \mathrm{l}$ Acetanhydrid versetzt und nach Versiegeln des Gläschens über Nacht im Trockenschrank bei $90^{\circ} \mathrm{C}$ temperiert. Danach wird wiederum im Stickstoffstrom bei $70^{\circ} \mathrm{C}$ zur Trockne eingedampft und der Rückstand mit $500 \mu \mathrm{l}$ Wasser und $100 \mu \mathrm{l}$ Chloroform geschüttelt. Die Chloroformphase wird in ein neues Injektionsgläschen gebracht, im Stickstoffstrom eingedampft und der Rückstand in $20 \mu \mathrm{l}$ Chloroform gelöst, das Gläschen versiegelt und davon ein Aliquot zur Injektion in den Gaschromatographen verwandt. Die Derivate sind bei Raumtemperatur wochenlang stabil.

\section{Gaschromatographie}

Wie bei Mcthode I

\section{Methode III}

Verfahren mit Methoximtrifluoracetaten als Derivate für Diund Trisaccharide

Bei der Trifluoracetylierung von Substanzen mit alkoholischen Hydroxylgruppen mittels Trifluoracetanhydrid entsteht Trifluoressigsäure. Da wegen der Hydrolyseempfindlichkeit der Derivate im Gegensatz zu Methode I und Il eine wäßrige Aufarbeitung nicht möglich ist, sondern wie bei der Trimethylsilylierung direkt aus dem Derivatisierungsansatz gespritzt werden muß, kann die Trifluoressigsäure nicht entfernt werden. Bei den zur Gaschromatographie von Disacchariden erforderlichen Temperaturen bewirkt aber diese starke Säure eine Zersetzung. Dies kann bei Verwendung des ebenfalls sehr reaktionsfreudigen N-Methyl-bis-(trifluoracetamid) als Derivatisicrungsmittel vermieden werden, da hierbei als Nebenprodukt bei der Veresterung das inerte $\mathrm{N}$-Methyltrifluoracetamid entsteht (7). Damit seitens der Carbonylfunktion keine Störung auftreten kann, wird diese mittels Methoxylaminhydrochlorid in Pyridin aufgehoben und daran anschließend im gleichen Ansatz die Veresterung mit N-Methyl-bis-(trifluoracetamid) durchgeführt. Die

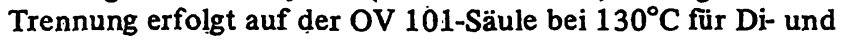
$160^{\circ} \mathrm{C}$ für Trisaccharide.

\section{Arbeitsvorschrift}

\section{Derivatisierung}

Von den nach der Methode von Somogyi (4) enteiweịten Zuckerlösungen werden $1 \mathrm{ml}$ zusammen mit $1 \mathrm{ml}$ Lösung des inneren Standards Triehälose der Konzentration 2,92 mmol/1 in einem Spitzkölbchen am Rotationsverdampfer zur Trockne eingedampft und anschließend mit $500 \mu l$ Methoxylaminhydrochlorid (Serva, Heidelberg) in Pyridin ( $10 \mathrm{~g} / 1$ Pyridin) versetzt und $1 \mathrm{~h}$ bei $70^{\circ} \mathrm{C}$ im Wasserbad temperiert. Die Lösung wird in ein Injektionsgläschen für die Gaschromatographie überführt und im Stickstoffstrom bei $70^{\circ} \mathrm{C}$ ungefähr zur Hälfte eingedampft. Nachdem $250 \mu \mathrm{l}$ N-Methyl-bis-(trifluoracetamid) zugesetzt sind, wird das Gläschen versiegelt und $5 \mathrm{~h}$ im Trockenschrank bei $70^{\circ} \mathrm{C}$ temperiert. Ein Aliquot des Derivatisierungsansatzes wird in den Gaschromatographen eingespritzt.
Gaschromatographie

Gerät:

Säule:

Trennflüssigkeit:

\section{PERKIN-ELMER F 11 (FID),}

Glas $6 \mathrm{ft}, 3 \mathrm{~mm} \phi$,

OV 101 (Applied Science Lab.) 3\% auf Gaschrom Q 100-120 mesh

(SERVA, Heidelberg),

Trägergas $\mathrm{N}_{2}$ : $30 \mathrm{ml} / \mathrm{min}, \mathrm{H}_{2}: 40 \mathrm{ml} / \mathrm{min}$, Luft: $400 \mathrm{ml} / \mathrm{min}$,

Säulentcmperatur: $\quad 130^{\circ} \mathrm{C}$ für $\mathrm{Di}-, 160^{\circ} \mathrm{C}$ für Trisaccharide,

Temperatur des

Injektionsblocks:

$160^{\circ} \mathrm{C}$ für $\mathrm{Di}-, 190^{\circ} \mathrm{C}$ für Trisaccharide, Temperatur des FID: $\quad 160^{\circ} \mathrm{C}$ für $\mathrm{Di}-, 190^{\circ} \mathrm{C}$ für Trisaccharide, Injektion:

Papiervorschub:

$1 \mu \mathrm{l}$,

$30 \mathrm{~cm} / \mathrm{h}$.

Dic Auswertung der Gaschromatogramme erfolgte manuell unter Verwendung des Ausdrucks ",Retentionszeit $\times$ Peakhöhe“ als Flächenmaß. Traten bei einem Zucker zwei Peaks auf, wurden diese getrennt ausgewertet und die Flächenmaße addiert.

Sämtliche Reagenzien waren, soweit nicht anders angegeben, Handelspräparate (p. a.) der Fa. MERCK, Darmstadt.

Für die statistischen Auswertungen verwendeten wir StandardRechenprogramme an einem Tischcomputer der Fa. DIEHL.

\section{Ergebnisse und Diskussion}

\section{Methode I (Methoximacetate)}

Nach dieser Derivatisierungsmethode gelingt die Trennung und quantitative Bestimmung von Maltose, Lactose und Saccharose auf einer OV 225 -Säule bei $260^{\circ} \mathrm{C}$. Lactose liefert zwei Peaks. Als geeigneter innerer Standard erweist sich Gentiobiose. Die Tabelle 1 enthält die absoluten und die auf Gentiobiose bezogenen relativen Retentionszeiten, während die Abbildung 1 ein entsprechendes Gaschromatogramm zeigt.

Tab. 1. Absolute und relative Retentionszeiten der peracetylierten Methoxime von Disacchariden (Methode I).

\begin{tabular}{lll}
\hline Disaccharid & $\begin{array}{l}\text { Retentionszeiten } \\
\text { absolut (min) }\end{array}$ & relativ \\
\hline Saccharose & 53,5 & 0,58 \\
Maltose & 58,5 & 0,64 \\
Lactose & 73,8 & 0,80 \\
(2 Peaks) & 78,0 & 0,84 \\
Gentiobiose & 92,4 & 1,00 \\
\hline
\end{tabular}

Diese Methode hat den Vorteil, daß die Derivatisierungsansätze ohne Gefahr der Hydrolyse aufgearbeitet, d. h. konzentriert werden können, was bei niedrigen Zuckerkonzentrationen in der Analysenlösung notwendig ist. Nach diesem Verfahren können Mono- und Disaccharide aus demselben Ansatz, wenn auch auf verschiedenen Säulen und bei verschiedenen Temperaturen, bestimmt werden (Monosaccharide: ECNSSM-Säule bei $190^{\circ} \mathrm{C}$, Disaccharide: OV 225 -Säule bei $260^{\circ} \mathrm{C}$ ). Die wichtigsten Disaccharide - Maltose, Lactose und Saccharose - 


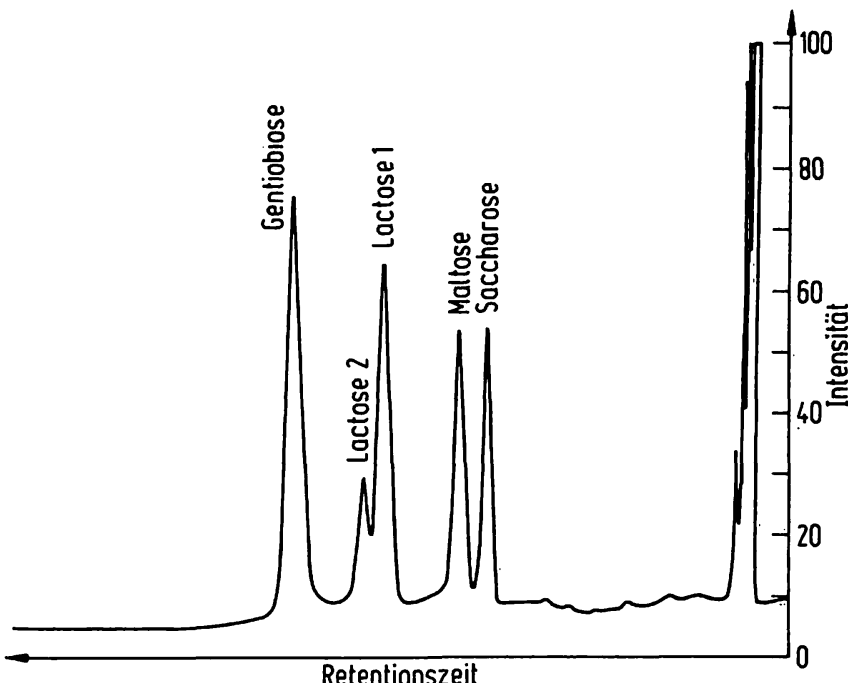

Abb. 1. Gaschromatographische Trennung von Disacchariden als octaacetylierte Methoxime (Maltose, Lactose) bzw. Octaacetat (Saccharose) (Methode I).

Lactose liefert 2 Peaks.

werden gut getrennt. Die Gaschromatogramme sind übersichtlich.

\section{Methode II (Alditolacetate)}

Auch hier ist eine Trennung und Bestimmung von Maltose, Lactose und Saccharose unter den gleichen Bedingungen wie bei Methode I möglich unter Verwendung von Trehalose als innerem Standard. Erwartungsgemäß liefert jeder Zucker nur einen einzelnen Peak. Da die Disaccharide Maltose und Lactose eine Carbonylgruppe enthalten, entstehen aus diesen infolge Reduktion der Carbonylgruppe zur alkoholischen Hydroxylgruppe Nonaacetate. Aufgrund dieses zusätzlichen Acetylrestes sind hier die Retentionszeiten für Maltose und Lactose unter gleichen Trennbedingungen im Vergleich zur Methode I wesentlich länger. In der Tabelle 2 sind die absoluten und die auf Trehalose bezogenen relativen Retentionszeiten zusammengestellt, die Abbildung 2 demonstriert die Trennung.

Methode II dürfte wohl die meisten Anwendungsmöglichkeiten bieten. Bei geeignetem Temperaturprogramm können aus dem gleichen Ansatz Monosaccharide, Ami-

Tab. 2. Absolute und relative Retentionszeiten der Alditolacetate von Disacchariden (Methode II).

\begin{tabular}{lcc}
\hline Disaccharid & $\begin{array}{l}\text { Retentionszeiten } \\
\text { absolut (min) }\end{array}$ & relativ \\
\hline Saccharose & 66 & 0,83 \\
Maltose & 105 & 1,34 \\
Lactose & 130 & 1,65 \\
Trehalose & 79 & 1,00 \\
\hline
\end{tabular}

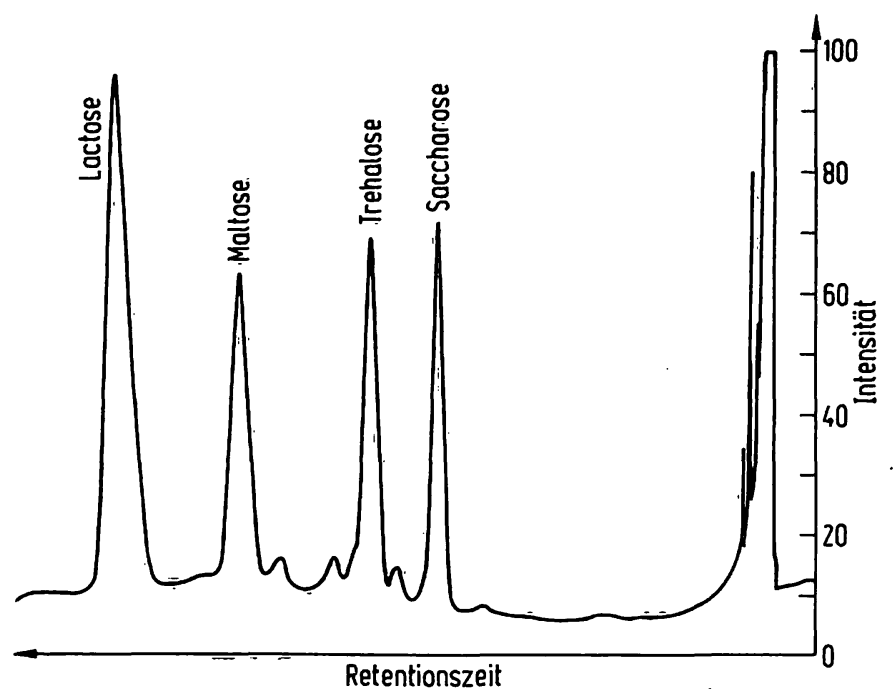

Abb. 2. Gaschromatographische Trennung von Disacchariden als Alditolacetate (Methode II).

nozucker und Disaccharide auf einer Säule und in einem Arbeitsgang getrennt werden. Die Derivate sind ebenfalls hydrolyseunempfindlich, können also durch Aufarbeitung konzentriert werden.

\section{Methode III (Methoxim-Trifluoracetate)}

Hier liefert Maltose zwei Peaks, von denen der spätere mit Lactose zusammenfallt. Zur Bestimmung von Maltose, Lactose und Saccharose eignet sich Trehalose und zur Bestimmung von Maltotriose, welche ebenfalls zwei Peaks ergibt, Raffinose als innerer Standard. Die entsprechenden absoluten und relativen Retentionszeiten sind der Tabelle $3 \mathrm{bzw}$. den Abbildungen 3 und $4 \mathrm{zu}$ entnehmen.

Tab. 3. Absolute und relative Retentionszeiten der pertrifluoracetylierten Methoxime von Di- und Trisacchariden (Methode III).

\begin{tabular}{lll}
\hline $\begin{array}{l}\text { Disaccharid } \\
\text { bzw. Trisaccharid }\end{array}$ & $\begin{array}{l}\text { Retentionsżeiten } \\
\text { absolut (min) }\end{array}$ & relativ (Trehalose) \\
\hline Saccharose & 29,2 & 0,64 \\
Maltose & 36,2 & 0,79 \\
(2 Peaks) & 43,4 & 0,95 \\
Lactose & 43,0 & 0,94 \\
Trehalose & 45,8 & 1,00 \\
Raffinose & 20,8 & - \\
Maltotriose & 41,4 & - \\
(2 Peaks) & 48,0 & \\
\hline
\end{tabular}

Die Methode III erlaubt zwar keine wäßrige Aufarbeitung und damit Konzentrierung des Dérivatisierungsansatzes, jedoch bedeutet das direkte Spritzen aus dem 


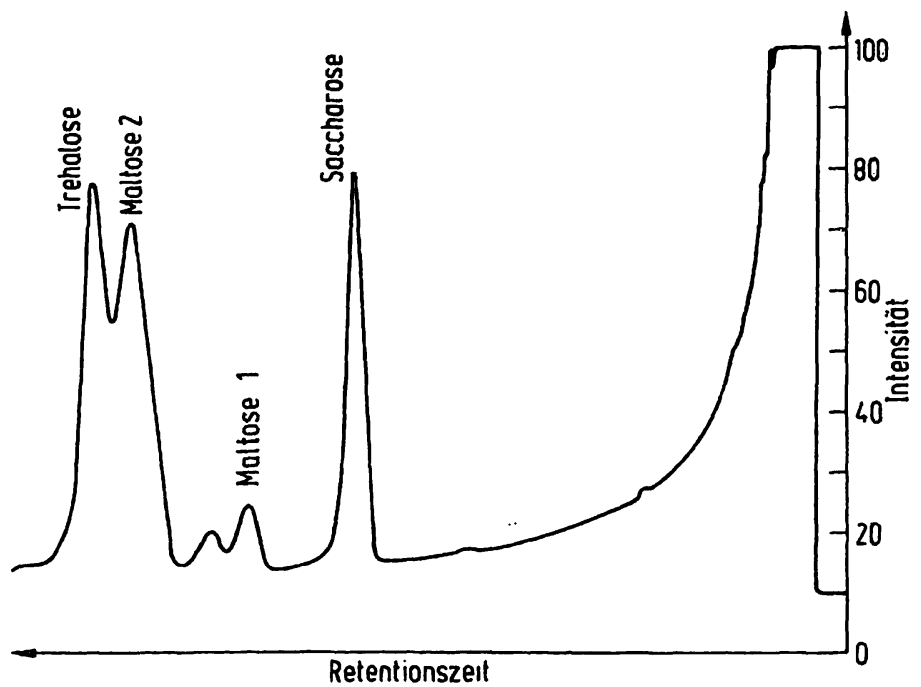

Abb. 3. Gaschromatographische Trennung von Disacchariden als octatrifluoracetyliertes Methoxim (Maltose) bzw. Octatrifluoracetate (Saccharose und Trehalosc) (Methode III).

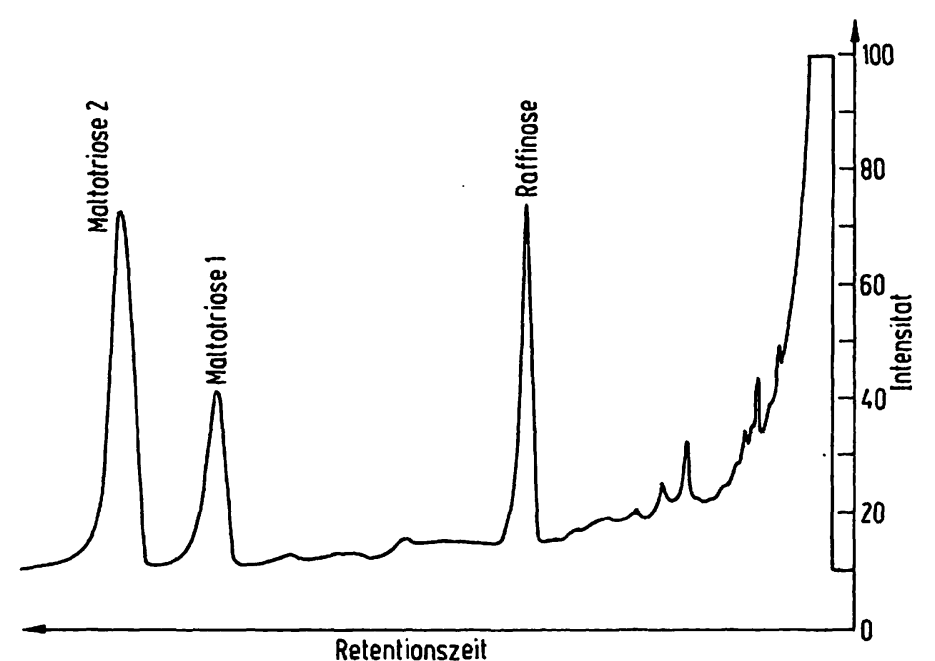

Abb. 4. Gaschromatographische Trennung von Trisacchariden als pertrifluoracetyliertes Methoxim (Maltotriose) bzw. Pertrifluoracetat (Raffinose) (Methode III). Maltotriose liefert 2 Peaks.

Ansatz eine Arbeitsersparnis. Andererseits kann aufgrund des Fluorgehalts der Derivate bei Verwendung eines Elektroneneinfangdetektors (ECD) zur spezifischen Detektion die Empfindlichkeit wesentlich gesteigert werden. Die Derivate sind im Vergleich zu den Acetaten derart flüchtig, daß bei relativ niedrigen Temperaturen

\section{Literatur}

1. Mehnert, H., Haslbeck, M. \& Förster, H. (1972), Dtsch. Med. Wochenschr. 97, 1763-1766.

2. Sweeley, C. C., Bentley, R., Makita, M. \& Wells, W. W. (1963), J. Amer. Chem. Soc. 85, 2497-2507.

3. Murphy, D. \& Pennock, C. A. (1972), Clin Chim. Acta 42 , $67-75$.

4. Somogyi, M. (1952), J. Biol. Chem. 195, 19-23. im Gegensatz zu den Methoden I und II sogar Trisaccharide getrennt werden können. Mit einem geeigneten Temperaturprogramm können also Mono-, Diund Trisaccharide aus dem selben Ansatz in einem Arbeitsgang bestimmt werden.

\section{Zuverlässigkeitskriterien der gaschromatogra-} phischen Zuckerbestimmung nach Methode I

Ausgehend von einer Stammlösung, die die Disaccharide Maltose, Lactose und Saccharose nebeneinander in der Konzentration 5,84 mmol/l enthielt, wurden durch Verdünnen auf die halbe Konzentration bis herab zur Grenzkonzentration $0,05 \mathrm{mmol} / 1$ acht Lösungen gewonnen, in welchen die genannten Disaccharide in jeweils gleicher Konzentration vorlagen. Aus solchen Lösungen wurden die Disaccharide quantitativ bestimmt und die Präzision in der Serie und von Tag zu Tag überprüft. Die Tabelle 4 enthält die Ergebnisse für die Konzentrationen 5,84 bzw. $0,05 \mathrm{mmol} / 1$ sowie für Wiederfindungsversuche, bei denen bekannte Mengen von Disacchariden eingewogen wurden. Die Zuverlässigkeit dieser Methode ist mit der von uns sehr gründlich untersuchten gaschromatographischen Bestimmung von Monosacchariden nach dem Methoximacetatverfahren vergleichbar.

Tab. 4. Zuverlässigkeitskriterien der gaschromatographischen Zuckerbestimmung (nach Methode I).

Es wurden wäßrige Lösungen von Lactose, Maltose und Saccharose in den angegebenen Konzentrationen verwendet.

\begin{tabular}{ll} 
Empfindlichkeit & $>0,05 \mathrm{mmol} / \mathrm{l}$ \\
Wiederfindung & $97 \pm 4,3 \%$ \\
$\begin{array}{l}\text { Präzision in der Serie } \\
\text { bei } 5,84 \mathrm{mmol} / \mathrm{l}:\end{array}$ & $\mathrm{VK}=3,2 \%$ \\
bei $0,05 \mathrm{mmo} / \mathrm{l}:$ & $\mathrm{VK}=15,0 \%$ \\
$\begin{array}{l}\text { Präzision von Tag zu Tag } \\
\text { bei } 5,84 \mathrm{mmol} / \mathrm{l} \\
\text { bei } 0,05 \mathrm{mmol} / \mathrm{l}\end{array}$ & $\mathrm{VK}=3,5 \%$ \\
\hline
\end{tabular}

\section{Danksagung}

Herr Prof. Dr. F. H. Schmidt, Leiter der Abt. Stoffwechselforschung BOEHRINGER-Mannheim, ermöglichte die Anfertigung von Elementaranalysen und Massenspektrogrammen der präparativ dargestellten Zuckerderivate, wofür auch an dieser Stelle herzlich gedankt sei.

Unseren Mitarbeiterinnen Frau B. Kössler und Frau A. Spieles sind wir für stets zuverlässige technische Assistenz zu Dank verpflichtet. Frau I. Kleßen danken wir für die sorgfältige Anfertigung des Manuskripts.
5. Yang, H.-J. \& Hakomori, S.-I. (1971), J. Biol. Chem. 246, 1192-1200.

6. Metz, J., Ebert, W. \& Weicker, H. (1971), Clin. Chim. Acta 34, $31-46$.

7. Donike, M. (1973), J. Chromatogr. 78, 273-279.
Prof. Dr. R. Kattermann Klinisch-Chemisches Institut, Klinikum Mannheim der Universität Heidelberg Postfach 23

D-6800 Mannheim 1 